\title{
APAMI
}

\section{Asia Pacific Association for Medical Informatics (APAMI)}

\author{
Naoki Nakashima, Rafiqul Islam Maruf, Louise Schaper, Chun Por Wong, Oommen John, \\ Shashi Bhushan Gogia, In Young Choi, Inga Hunter, Alvin Marcelo, Ming-Chin (Mark) Lin, \\ Boonchai Kijsanayotin and Vajira H. W. Dissanayake
}

\section{APAMI Membership}

APAMI's current, active eleven member societies include (1) the Health Informatics Society of Australia (HISA), (2) the Hong Kong Society of Medical Informatics (HKSMI), (3) the Indian Association for Medical Informatics (IAMI), (4) the Japan Association for Medical Informatics (JAMI), (5) the Korean Society of Medical Informatics (KOSMI), (6) the Health Informatics New Zealand (HiNZ), (7) the Philippine Medical Informatics Society (PMIS), (8) the Health Informatics Society of Sri Lanka (HISSL), (9) the Association for Medical \& Bioinformatics Singapore (AMBIS), (10) the Taiwan Association for Medical Informatics (TAMI), and (11) the Thai Medical Informatics Association (TMIA). APAMI has a number of observer members including societies from Bangladesh, Indonesia, Malaysia, and Nepal. Although Kazakhstan, Pakistan, and Vietnam had APAMI member societies in the past, APAMI leadership has been unable to elicit any updates from these member societies.

\section{APAMI Executive}

The current APAMI leadership consists of President: Naoki Nakashima, Japan Association for Medical Informatics (JAMI) [nnaoki@med.kyushu-u.ac.jp]; General Secretary: Ming-Chin (Mark) Lin, Taiwan Association for Medical Informatics (TAMI) [arbiterski@gmail.com]; Treasurer: CP Wong, Hong Kong Society of Medical Informatics (HKSMI) [cp@cpwong. com] Immediate Past Executive Committee: President: Vajira H. W. Dissanayake, Health Informatics Society of Sri Lanka (HISSL) [vajira@anat.cmb.ac.lk]; General Secretary: Dr. Oommen John, Indian Association for Medical Informatics (IAMI) [ojohn@georgeinstitute. org.in]; Treasurer: CP Wong (HKSMI)[cp@cpwong. com]

\section{APAMI Website}

Current APAMI website:

http://www.apami.online

\section{APAMI 2020 Activities}

While APAMI activities in 2020 were restricted by the COVID-19 pandemic, APAMI secretariat facilitated a number of engagements in the region. The World Health Organization invited APAMI President to be one of the speakers at the launch of the Digital Investment Implementation Guide on 14 October 2020. APAMI President and Secretary presented about the informatics initiatives for COVID19 pandemic response in the APAMI region during the American Telemedicine Association Conference. An APAMI regional informatics best practices during the COVID19 pandemic was presented as a lightning talk at the 2020 Global Digital Development Forum.

APAMI also conducted the scheduled biennial conference successfully in Japan on November 2020 in spite of COVID-19 pandemic in a hybrid (with both online and on-site participation) system. There was a General Assembly (GA) meeting in the final day of the conference. Almost all members joined in this GA and discussed on the annual financial statement, last year activities and future directions. This GA also officially selected Dr. Ming-Chin (Mark) Lin (Taiwan) as the General Secretary and Dr. CP Wong (Hong Kong) as the Treasurer of APAMI for the year of 20212022 under the presidency of Professor Naoki Nakashima (Japan).

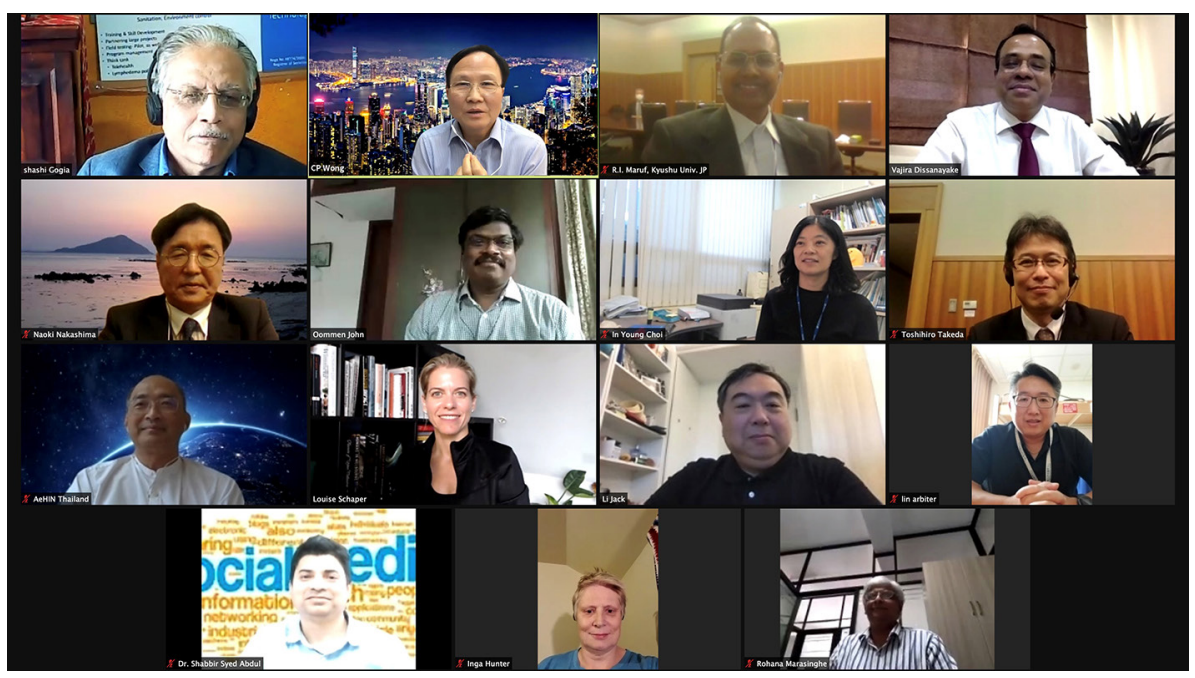

Fig. 1 The General Assembly (GA) Meeting of APAMI held on November 23, 2020. 


\section{APAMI 2020 Conference (APAMI2020, Hamamatsu, Japan)}

The 11th Biennial Conference of Asia-Pacific Association of Medical Informatics (APAMI2020) was held on November 2123, 2020 in Hamamatsu, Japan. This hybrid conference (both online and online) was hosted by the Japan Association of Medical Informatics (JAMI). The JAMI president Naoki Nakashima was the General Chair of this conference while Rafiqul Islam Maruf of Kyushu University and Toshihiro Takeda of Osaka University were the Chairs of the Organizing Committee and the Scientific Program Committee respectively. The theme of this conference was "Informatics for Patient Engagement". Besides, COVID-19 was set as a special topic in this conference due to the present pandemic situation. In this conference, there were 5 keynote speeches from the renown experts of Medical Informatics. They were (1) Prof. William Ed Hammond of Duke University, USA on "All About FHIR", (2) Dr. Clement J McDonald of National Library of Medicine, USA on "Tools for Validating, Entering and Retrieving FHIR Clinical Data from the Lister Hill Center FHIR Brigade", (3) Prof. Hyeoun-Ae Park of Seoul National University, South Korea on "Role of Health Informatics for Patient Engagement", (4) Prof. Naoki Nakashima on "Patient Engagement and PHR" and (5) Prof. Christopher G. Chute of Johns Hopkins University, USA on "The Potential Roles of ICD-11 in Translational Research- Architectural Features that Support Data Science". There were 2 APAMI Panel sessions. The first panel session was on "Patient Engagement" moderated by Dr. Shabbir Syed-Abdul (Taipei Medical University, Taiwan), and the panel speakers were Dr. Luis Fernandez Luque (Salumedia Tecnologias, Sevilla, Spain \& Adhera Health, USA), Dr. Ekaterina Koledova (Affiliation: Merck Healthcare KGaA, Frankfurter Str. 250, 64293 Darmstadt, Germany), Dr. Rafiqul Islam Maruf and Prof. Jack Li (Taipei Medical University, Taiwan). The second panel session was on "COVID-19" jointly moderated by Prof. Vajira HW Dissanayake and Dr. John Oommen, and the panel speakers were Dr. Mark Landry (Department of Health Systems Development, Regional Office for South-East Asia, World Health Organization, New Delhi,
India), Dr. Louise K Schaper (Australasian Institute of Digital Health, Melbourne, Australia) and Prof. Siaw-Teng Liaw (School of Public Health and Community Medicine, UNSW Sydney, Australia). Besides, there were 2 workshops on "On Open Access Publishing in Medical Informatics" moderated by Reinhold Haux (Peter L. Reichertz Institute for Medical Informatics of TU Braunschweig and Hannover Medical School, Germany) and "Publish or Perish: Introducing the New BMJ Journal for Informatics" moderated by Prof. Jack Li. Although the COVID-19 pandemic created a big obstacle for joining to this hybrid conference, there were more than 220 participants from 22 countries around the world.
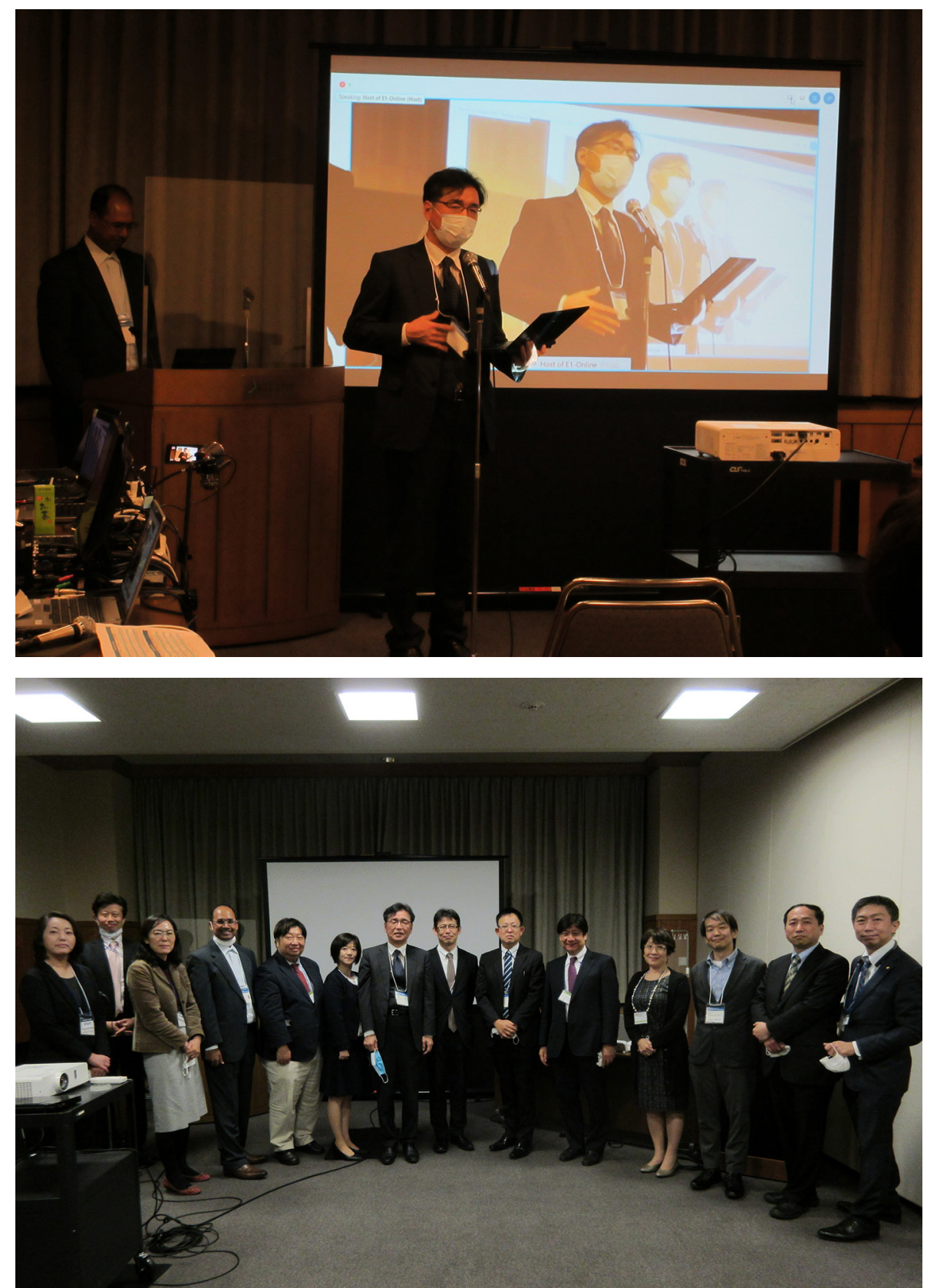

Fig. 2 The General Chair of the APAMI2020 Prof. Naoki Nakashima and the Conference Committee Members including Prof. Michio Kimura during the Conference in Hamamatsu, Japan. 


\section{APAMI Publishing Journals}

The Journal of Computer Methods and Programs in Biomedicine (CMPB) has been attached with APAMI as an official journal. The CMPB will arrange a peer-review process of the selected 13 best papers of APAMI2020 conference and issue a special APAMI2020 Edition with the successfully reviewed papers. However, it is a regular journal but not open. The APAMI General Assembly Meeting 2020 has agreed to make BMJ Health \& Care Informatics (BMJ HCI) as the 2 nd official journal of APAMI. This is an open access journal and Prof. Jack Li is the Editor-in-Chief.

\section{Reports of APAMI Member Societies}

\section{[1] Health Informatics Society of Australia}

2020. We survived, and we're exhausted. Is that ok? Is that enough? .... No? You want to know more? Ok, well here goes. :-)

2020 started with a 'bang' for health informatics and digital health in Australia. The signature line in our emails literally was a fireworks GIF. In December 2019, following 2 years of consultation with members, Fellows and stakeholders, members of the Health Informatics Society of Australia (HISA) and the Australasian College of Health Informatics (ACHI) voted in favour of merging the organisations to form the Australasian Institute of Digital Health (AIDH or the 'Institute').

Christmas was cancelled and the staff worked exceptionally hard to have everything ready to launch the Institute on 24 February 2020. We had a launch party to celebrate. We had t-shirts made, we danced, we drunk, we ate. The Australian digital health community were excited about the Institute's strategy and were wanting to be involved in our grand adventure. At the time, COVID19 was a relatively small problem confined to China and the Australian government had closed our borders to China as a temporary measure, while we all marvelled at how Wuhan, a city in our region could 'shut down'. It was a simpler, more innocent time that now, 12 months later, seems like a lifetime ago.

Energised by the mandate members and Fellows gave us, we had big plans for 2020; but 3 weeks after the launch we were facing a difficult reality - one which defined 2020 for all of us, particularly those in Melbourne,
Victoria. A State of Emergency was declared and the Australian government mandated that all non-essential services and business would temporarily be shut down. Three weeks after our launch, we shut down our office and all staff were required to work from home. At the time we were hoping to return to the office 6-8 weeks later and instead we spent all of 2020 working from home. The tight-knit team that runs the Institute didn't see each other in person for almost 10 months. A second-wave outbreak in Victoria effectively shut down the State and we spent months living under a restrictive and long-lasting lockdown which limited movement outside the home to 1 hour per day.

2020 for the Institute, did end up being defined by reinvention, just not of the sort we had originally planned for.

Faced with an uncertain future, our reality might be very different now to how we envisaged it back in February 2020, but the Institute is thriving thanks to the loyalty and commitment of our members and the individuals and organisations that make up our community; and thanks to the vision of the Board; and the creativity, energy and innovativeness of our staff.

With in-person gatherings banned, the AIDH team taught themselves how to run high quality, virtual events at scale and developed multiple new event series to maximise value to members and give the community networking and professional development opportunities. We ran weekly virtual events - Digital Health Live for 1 hour of professional development with a guest speaker(s) and \#ZoomBar a 1 hour informal catch-up with invited guests to share a beverage and chat all things digital health. Our new virtual conference From the Frontlines ran twice due to its popularity, and focused on COVID19 related digital health efforts.

Similar to many membership bodies, our annual series of conferences and events, generates the majority of our revenue. The loss of this income stream was (and remains) a significant blow to the organisation. In order to address this revenue gap, we developed a new national event, the Digital Health Institute Summit. With all our data models from previous years no longer valid, we had no idea if this new event would address what members wanted and if they would support it by speaking and attending. To our surprise, our concerns were unfounded. The Summit ended up being the largest digital health event ever ran in Australia, with 300+ speakers, 17 days of programming and so much content that the month of November became Digital Health Month, with every day devoted to a different digital health theme, with significant virtual engagement plus smaller in-person gatherings in 3 capital cities.

Our plans to host IMIA's Nursing Informatics 2020 Congress, to coincide with the International Year of the Nurse were also thwarted by the global pandemic. Plans are currently underway to host IMIA NI in 2021 which will be virtual for those unable to travel to Brisbane. We are so very grateful to the international community and IMIA for their support and flexibility in accommodating the need to change the timing and the method of delivery for IMIA NI; and likewise to IMIA and Taiwan for working with us to delay our hosting of MedInfo, which will be held in Sydney in 2023.

The Institute has at its core a commitment to build digital health capability across the healthcare workforce. Due to the pandemic, we paused a number of workforce initiatives that were to launch in 2020 which will now be a focus of 2021. While we anticipated a drop in enrolments in our certification program due to a shift in priorities of the healthcare sector, CHIA (Certified Health Informatician Australasia) reached a new milestone with over 1000 individuals certified in health informatics. Behind the scenes, the committees were incredibly busy and completed a comprehensive review of Australia's Health Informatics Competency Framework and the CHIA exam - working collaboratively via Zoom on workshops held on Saturdays. CHIA "2.0" will launch in 2021.

We launched two new pathways to Fellowship of the Institute. One is focused on $\mathrm{PhD}$ students who undertake a program of learning and work experience to be "fast-tracked' to Fellowship. There are currently 16 students in our Fellowship by Training program, with 3 who have graduated from the program. The other pathway is for Digital Health Executives, whose management and executive experience is recognised by AIDH by awarding them Fellow status. We held a joint event for executives with Digital Health 
Canada, which was appreciated and valued by our newly minted Fellows. We currently have over 200 Fellows and Associate Fellows.

In brief - we also launched a white paper entitled What is Digital Health? And Why Does It Matter? which has received international recognition. Led by members, we developed, consulted and launched 10 Principles for the Australian Government's COVID19 Contact Tracing App. We worked with government and industry stakeholders to progress the development of Standards and their selection in Australia. We also worked with the Australian government and nursing and midwifery bodies and professionals to develop and launch the National Nursing and Midwifery Digital Health Capability Framework. This work has also received national and international recognition.

Finally, we launched InGeNA, the Industry Genomics Network Alliance. InGeNA is supported by $>15$ industry bodies and organisations along the value chain of genomics and digital health. The aim of InGeNA is to integrate genomics into healthcare. We expect that will take us a few years! :-3)

While we had a successful 2020, 2021 will continue to pose challenges for the Institute due to the disruption to our traditional revenue streams making it difficult to meet the rising expectations of our members and the health community. We will meet these challenges by continuing to evolve and by putting our people at the heart of everything we do.

We send our condolences to all who have lost someone, who are dealing with ongoing personal and professional hardships. We wish our IMIA friends and colleagues all the best for 2021 and look forward to being able to see you, in-person, in 2023. In the meantime, we also send you virtual, socially distanced hugs.

\section{[2] Hong Kong Society of Medical Informatics}

In the last one year amidst the social movement and pandemic COVID-19, the activities of the HKSMI has been severely hampered. Yet, we are very lucky in that we still manage to hold a very successful WHO Global Digital Health Partnership Summit cum the Asia Pacific Electronic Health Records Conference in October 2019. A total of more than 800 participants from 40 countries came to attend despite the political unrest during that period. We are most grateful for their support.
The Hong Kong eHealth innovation, however, continues to evolve into Phase II of EHR sharing system platform which will include sharing of radiology images between the public and private sectors, in addition to the 15 years long sharing of radiological reports across the 2 sectors. Patient Portals and incorporation of Chinese Medicine in the sharing platform is under way.

We are still able to hold a few seminars via webinar style and continue to keep the momentum of eHealth development moving in Hong Kong.

\section{[3] Indian Association for Medical Informatics (IAMI)}

The Ministry of Health and Family Welfare, Government of India hosted an expert consultation on the National Digital Health Blueprint, our members were actively engaged in supporting the Ministry of health in collating and consolidating the technical inputs to ensure compliance to the best practices and evidence-based approaches to establishing the national digital health infrastructure. Further, few members were actively engaged during the stakeholder's consultation on the National Digital Health Mission by the National Health Authority, Government of India. The NDHM is the nodal agency that is implementing the national Health ID that aims to help create longitudinal health records for the citizens. The role of health informatics for the operationalization of the insurance led model under Ayushman Bharat (PM-JAY) program towards universal health coverage to improve access to healthcare was highlighted during the consultations and through publications by the members.

Several partnerships with several professional societies and International associations leading a strong advocacy for bringing evidence-based informatics practices on the strategic initiatives being rolled out both in India and the APAMI region were also undertaken during 2020 despite the challenges posed by the pandemic. Our members in close partnership with WHO South East Asia Regional Office facilitated virtual consultations on the telemedicine practice guidelines issued by Indian Government during the COVID-19 pandemic, these consultations led to wide spread policy advocacy on embedding electronic health records into teleconsultations to ensure the continuum of care and transparency on quality of care provided through telemedicine.

Our members continue to play key and integral role at the subcommittee on health informatics standards at the Bureau of Indian Standards, Government of India A new subgroup on telehealth standards has been established during the COVID-19 pandemic and our members are leading a separate thematic scoping of telehealth standards for improved and standardized clinician patient communications leveraging innovative technology tools is underway and has been well received by all the stakeholders at Bureau of Indian Standards. Two recent initiatives from India as P member of ISO TC215 need wider dissemination in the hope of getting international participation from the IMIA Fraternity.

Health Informatics usage and related standards are helping further adoption of Indian Traditional medicines especially Ayurveda, which is excellent for lifestyle management. Its propagation of healthy diets and herbs has been appreciated in fighting COVID in India.

Creating standardized Icons and Graphics for usage within health applications. These will hopefully assist in allowing better understanding and adoption of available standards like SNOMED CT.

\section{[4] Japan Association for Medical Informatics (JAMI)}

As of December 31, 2020, JAMI has more than 3,800 members consisting of about 2,540 regular members, 380 affiliate members, and more than 880 members in other categories. Since 2019, the president Professor Naoki Nakashima has been leading the board members. JAMI has tried new style of academic congresses in 2020 due to COVID-19 pandemic. The Annual Spring Conference was held on June 5-6, 2020 as a live online conference. The theme of the conference was "What and How Should We Share in Medical Informatics?". More than 2,300 attendees from JAMI and other societies remotely participated by online system and discussed on various hot topics in biomedical informatics. The 40th Joint Conference on Medical Informatics 
(JCMI40) was held on November 18-22, 2020 in Hamamatsu city of Shizuoka prefecture as a hybrid conference (both online and onsite). More than 2,900 attendees joined to this conference. At the same time, JAMI also hosted the APAMI2020 conference in the same venue as a parallel hybrid event on November 21-23, 2020. The slogan of the APAMI conference this time was "Informatics for Patient Engagement". In APAMI2020, there were 70 international attendees from all over the world in additional to 150 or more attendees from JCMI40. Regarding JAMI Healthcare Information Technologists, this is unfortunate that we had to cancel the certification examination in 2020 due to COVID-19 situation. JAMI established a task force team to respond "with COVID-19 era" and proposed three strategies. In "Continuation of Discussion", JAMI repeated to have various sessions of "Medical Informatics in with COVID-19 era" in the annual spring conference and JCMI40. In "Information Sharing", JAMI posted varieties of the standard codes about COVID-19 on the JAMI website. In "Social Contribution", JAMI accepted a donation of equipment for remote conference from a company and spread them to 40 hospitals.

\section{[5] Korean Society of Medical Informatics (KOSMI)}

South Korea announced the amendments of the Personal Information Protection Act("PIPA") to develop a 'data economy' by introducing the concept of 'pseudonymised data' and a legal basis upon which data may be utilized more flexibly (to an extent reasonably related to the original purpose of collection). Under the amended PIPA, a personal data controller will be permitted to use and release personal data without obtaining the consent of the data subject after considering whether the data subject's rights would be infringed upon and/or measures to secure the integrity of the personal information have been properly taken. The Personal Information Protection Commission (the "PIPC") also published its "Comprehensive Guidelines on Processing Psedonymized Data". In addition, Ministry of Health and Welfare(MOHW) and PIPC published the specific guidelines on de-identification process and utilization of healthcare data.
As of December 31, 2020, KOSMI has more than 8599 different types of members consisting of 239 life time members, 232 full members, 36 student members, 8087 Web members, and 5 institute members. KOSMI is organizing biannual academic conferences, seminars on different topics, and publishing the Health Informatics Research (HIR), an official journal of KOSMI four times per year.

The spring conference could not be held Due to COVID-19 this year. The fall conference was held at Siheung Camputs of Seould National University from November 12 to November 13.

Keynote speakers are Sungrho Yoon from Presidential Committee on The Fourth Industrial Revolution, and Geun-chan Lim from Korea Health Information Service. Totally, 550 members participated in the conference.

There were 2 tutorials, 18 symposiums, 18 paper presentations, and 41 poster presentations. Four papers, three posters were awarded and recognized as the best papers and poster respectively at the conference. Also, BIT medical information academy prize by BIT computer company also awarded.

KOSMI published 26th volume of the Health Information Research (HIR) in 2020. The HIR publishes four issues in English including 4 editorials, 1 review articles, 31 original research articles, 4 case report, 1 Tutorials, 3 communications, and 1 erratum. The Journal was accredited by the Korea Research Foundation in 2006 and registered as one of prestigious academic journals in Korea.

\section{[6] Health Informatics New Zealand (HiNZ)}

In March 2020, New Zealand entered full lockdown (alert level 4) with $80 \%$ of consultations moving to telehealth. The lockdown led to the cancellation of Health Informatics New Zealand's (HiNZ) two regular conferences. After considering the costs/risks of replacing them with virtual or hybrid events, HiNZ decided to go ahead with weekly webinars instead. This was well received and supported by the sector, achieving excellent viewer numbers (live and on-demand views) and had good sponsorship support. The intent is to continue running webinars every second week (sometimes weekly) throughout 2021.
HiNZ ran the first in-person events in late November 2020 as 100-person regional networking events, as these were low risk, as they would be able to run even if there were social distancing requirements in place. 200-person regional events are scheduled for March 2021 and HiNZ hopes to run the first large scale national event, for 1400 delegates, in Wellington in November 2021.

During the pandemic, HiNZ's digital magazine eHealthNews.nz, carried a lot of COVID-19 related news items and sector announcements during the April - June 2020 period. It was perceived by the sector as a trusted source of New Zealand based digital health news and provided a useful service.

The New Zealand Telehealth Leaders Group likewise ran very successful webinars for the health sector and provided support for health providers and consumers on the use of telehealth for consultations. Several research projects are underway to maintain the investment in telehealth.

\section{[7] Philippine Medical Informatics Society (PMIS)}

On November 9, 2019, the Philippine Medical Informatics Society (PMIS) with support from the University of the Philippines Manila - Medical Informatics Unit co-organized the Electronic Health Records (EHR) Summit at the Philippine Heart Center. With more than two hundred guests, the one-day event featured leaders from the public and private sector discussing current trends and challenges with digital health from their respective agencies. Foremost in the exchanges was the importance of governance in managing the complexity of digital health design and implementation. In the context of the newly passed Universal Health Coverage law, it is anticipated that digital health will be one of the levers by which UHC can be achieved.

The year 2020 was marked by the emergence of the coronavirus pandemic. While almost all sectors of society ground to a halt, health informatics activities were at a flurry having been one of the sectors that had been regularly holding webinars even before the crisis. PMIS leaders were invited to give lectures and participate in online panels. Health IT applications such as telemedicine platforms and electronic medical records proliferated the health sector. Specialized applications 
such as contract tracing were submitted for consideration by a government-led national inter-agency task force. Amidst all of these, PMIS leaders led the development of telemedicine guidelines which became a resource for the Department of Health in their issuance to all telehealth practitioners in the country.

\section{[8] Health Informatics Society of Sri Lanka (HISSL)}

2020 was a challenging year for HISSL. The usual activities of HISSL were curtailed and the members of HISSL focused their attention on the digital response to the COVID-19 pandemic. In January of 2020, foreseeing the need for COVID-19 surveillance, HISSL partnered with the Ministry of Health Sri Lanka to customize the DHIS2 tracker for COVID-19 case detection. The DHIS2 COVID-19 package that was inspired by this design is now deployed in over 50 countries around the world (https://www.dhis2.org/ covid-19). HISSL also partnered with the Commonwealth Centre for Digital Health (CWCDH) to implement the SelfShield app that was designed, by the CWCDH innovation team based in Sri Lanka, to support self-health checks by individuals and the management of quarantine and self-isolation programs managed by health institutions in Sri Lanka.

HISSL as the main technical partner of the CWCDH provided technical support for the Virtual Commonwealth Civil Society Policy Forum held on 6 May 2020 on "Strengthening Health Systems with Digital Technologies in Midst of the COVID-19 Pandemic to Ensure Universal Health Coverage" and the 4th Commonwealth Digital Health Awards that culminated with the awards ceremony on 28 November 2020. HISSL managed the judging process and hosted the awards ceremony of the Commonwealth Digital Health Awards. It attracted nearly 100 applicants from more than 25 countries. The entire process was managed through virtual platforms including the judging process which consisted of a live and offline sessions contributed to by a 30 strong panel of judges drawn from across the world.

The HISSL is also the main technical partner of the Ministry of Health Sri Lanka on digital health. Medical Education and Digital Health experts from HISSL took part in a collaborative project with the World
Health Organization (WHO) and the Ministry of Health in developing and launching the Non-Communicable Diseases Learning Platform in Sri Lanka. The learning management system is now capable of providing distance training on NCD care for health workers around the country in the most efficient manner. Building on this experience, HISSL supported the Faculty of Medicine, University of Colombo to introduce several new changes to the teaching and learning process at the beginning of the pandemic. This helped the faculty to minimize the impact of the lock down and social distancing and to continue the teaching activities online.

In addition to the Non-Communicable Diseases Learning Platform, HISSL is supporting WHO in Sri Lanka to develop an image database of food items to track the color code introduced by the Ministry of Health to sensitize the general public on the sugar, salt and fat contents of the processed food varieties.

HISSL also plays a significant role in multiple collaborative projects in partnership with international agencies such as Vital Strategies, WHO, CDC foundation, AeHIN and Data for Health initiative. The focus areas of these projects included medical certification of cause of death, digital health atlas, open source Insurance Management Information System (OpenIMIS), COVID-19, Non-Communicable Diseases Survey and Salt reduction.

The Medical Certification of the Cause of Death project is now ready to introduce a curriculum to introduce medical certification of death to the undergraduate medical curriculum in technical partnership with the University of Melbourne. Under the OpenIMIS project, HISSL has initiated a broader dialog with insurance providers in Sri Lanka, both state owned and private sector, to understand their information requirement for the policy formulation in life and health insurance sectors. This project also expects to support the role played by health insurance towards the universal health coverage.

\section{[9] Taiwan Association for Medical Infor- matics (TAMI)}

The Taiwan Association for Medical Informatics (TAMI) supports research based on medical informatics, improving the quality of medical information, and augmenting international relations in the medical community. TAMI has nearly 300 active members, including clinical workers, information technology office members, academic researchers, and industrial engineers. TAMI contributes to enhancing communication and collaboration with international medical organizations, as well as promoting the exchange of a wide spectrum of medical information in Taiwan.

Events that took place in 2020 were as follows:

1. The Joint Conference on Medical Informatics in Taiwan (JCMIT) was organized by TAMI, National Taipei University of Nursing and Health Sciences, on August 24th and 30th, 2020. Because of the COVID-19 pandemic, we altered the conference format to a virtual meeting. The main topic of the conference was "Applying Information and Communication Technology to Realize Digital Health" and it was attended by medical experts, researchers, and scholars from various countries with whom we discussed issues in medical information systems.

2. TAMI continuously holds licensing examinations for assessment-based certification. We provide Medical Informatics Manager and Analyst Certificates for participants who meet the standards of knowledge of health information technology and management of the complex social organizational issues surrounding it. In 2020, we provided 225 and 458 such certificates, respectively, with a total pass rate of $65.4 \%$.

3. We are going to host the 20th World Congress on Medical and Health Informatics, MedInfo 2025, in Taipei, Taiwan. Therefore, we will be ready to integrate advanced networks, assemble patient data, and promote the development of telehealth to demonstrate Taiwan's achievements in Smart Medicine and eHealth.

4. The 2020 HealthCare Expo Taiwan was held at Taipei Nangang Exhibition Center, showcasing Asia healthcare transformation and unprecedented opportunities and solutions in medicine and healthcare in 2020. The President of the International Medical Informatics Association, Prof. Yu-Chuan Jack Li, was also presented 
the AI Industry Leadership Forum on how AI can help to monitor the trajectories of both in- and outpatients and make medicine more preemptive in terms of Earlier Medicine (patient safety and prevention) in the project.

\section{[10] Thai Medical Informatics Association (TMIA)}

Amid of the COVID 19 pandemic in 2020, TMI can organize our regular in-person annual conference during 25-27 November 2020. After flattening the curve of the first wave of the COVID-19 spreading in Thailand in May 2020, Thai government allowed public activities with mask waring, sanitation, and social distancing measures. The theme of the 2020 TMI annual conference was "Digital Health: New Normal". Although the limitation of allowed number participants, the conference was a success. The deputy minister of Ministry of Public Health, Dr. Satit Pitutacha, came and gave the keynote speech on Thailand's digital health transformation. There were 288 participants from all provinces across Thailand, $43 \%$ were clinicians (doctor/ nurse), $24 \%$ were IT professionals and $17 \%$ were health management information system officers and coders. TMI could also provide several in-person workshops and training before and after the first COVID 19 spreading wave (March to May 2020). They are basic and advance healthcare analytics workshops, mobile application development training, health IT project management workshops and the basic ICD 10 training and workshop.

In 2019, TMI has elected our first female president, Dr. Chamaree Chuapetcharasopon. Dr. Chamaree Chuapetcharasopon is a renowned physician, radiologist, and biomedical informatics professionals in Thailand. She has held leading role in many Thai medical, radiological, and biomedical informatics organizations including the pre- sident of Thai Medical Women's Association (under The Royal Patronage of Her Majesty the Queen), the president of Radiological Society of Thailand before accepting the TMI presidential role. The TMI members are honored that our former president, Dr. Choosna Makarasarn, accepted the association's President Emeritus and continue to provide advices to the association. In 2020, Dr. Boonchai Kijsanayotin, the TMI's vice president was inducted to become a fellow of the IMIA's International Academy of Health Science and Informatics (IAHSI). It is an honor to the awardee and the association.

TMI has played a crucial role in the field of biomedical and health informatics in Thailand by working with both local and international health communities and industries for sharing and exchanging news, information and knowledge in the field. As of December 2020, TMI has 510 registered members and around $50 \%$ are health care professionals. 